\title{
Estado del Arte sobre Concepciones de la Diversidad en el Contexto Escolar Infantil
}

\author{
State of Art about Conceptions of Diversity in Childhood \\ Education
}

\author{
Angélica María Barrero ${ }^{1 *}$ \\ Ana Lucia Rosero ${ }^{2}$ \\ ${ }^{1}$ Centro Neuro Psicopedagógico Sentir \& Pensar, Colombia \\ ${ }^{2}$ Universidad de San Buenaventura Cali, Colombia
}

\begin{abstract}
El presente artículo hace parte de la revisión documental Concepciones de los Educadores con relación a la diversidad y su incidencia en el aprendizaje infantil. El diseño de la investigación es de tipo cualitativo-interpretativo, de tipo documental. El propósito fue constatar qué y cómo se está trabajando la diversidad en el campo de la educación inicial, desde las concepciones de los educadores, entre los años 2000 y 2015, para ello se seleccionaron 41 investigaciones y se establecieron unas categorías de análisis. En los resultados emergieron 3 focos: Atribuciones de la diversidad, Aspectos metodológicos de la investigación y Denominaciones sobre el acto de pensar de los educadores sobre la diversidad; de igual forma en cada foco se establecen unas tendencias que permiten dar cuenta del propósito de la investigación.
\end{abstract}

Descriptores: Aprendizaje; Estudiantes; Educación de la primera infância; Diversidad cultural; Colombia.

\begin{abstract}
This article addresses the revision from Teachers 'conceptions about diversity and incidence in childhood learning. This investigation had the purpose of demonstrating the "what" and the "how" of diversity as being performed on the educational field from the conceptions of childhood educators. These facts were selected between the years of 2000 and 2015. There were 41 investigations. As a result, 3 areas of focus emerged: Diversity acknowledgments, Methodologic aspects of the research, and standards of thoughtful action of educators in relation to diversity. In addition, each area has established tendencies that allowed us to find the purpose of the investigation. The revision of the documents also permitted to understand the high percentage of diversity that continues to be stigmatized, and even assumed as a threat, resulting of discrimination and rejection of boys and girls which remains today as a daily practice.
\end{abstract}

Keywords: Learning; Students; Early childhood education; Cultural diversity, Colombia.

\section{Revisión de la literatura}

El presente artículo aborda la revisión documental a partir del tema de investigación Concepciones de los Educadores con relación a la Diversidad y su incidencia en el aprendizaje infantil. Esta revisión ha permitido dar cuenta que es un campo reciente de estudio, cuya relevancia comienza a tomar fuerza a partir de las declaraciones internacionales sobre la importancia de la visibilización de la Primera Infancia y sus

*Contacto: anmaba2010@hotmail.com

Recibido: 06/06/2017

ISSN: 0718-7378

$1^{a}$ Evaluación: 15/01/2018

www.rinace.net/rlei/

Aceptado: 28/02/2018 
particularidades, como población de derechos y de la trascendencia que tiene el educador en esta etapa de la vida.

Para Gimeno (2000), la diversidad es la característica de ser únicos y diferentes. De ahí que Parrilla (2006) refiera que la educación inclusiva, aquella que atiende la diversidad en el aula, no responda solamente a las necesidades de cierta población educativa, sino que atienda a todos los estudiantes. En este sentido, se esperaría que una institución abierta a la diversidad no tendría que hacer énfasis en la atención de algunas poblaciones. No obstante, se ha encontrado que la población a la que se ha relacionado con ser diversa es aquella que se considera vulnerable, es decir, que está atravesada por una condición de discapacidad, desplazamiento, origen étnico, etc.; lo que llevaría a que solo estudiantes que estén en dichas condiciones puedan ser visibilizados por ser diversos y así dar cumplimiento a las normativas educativas.

Todo lo anterior, lleva a preguntarse, ¿qué lugar ocupan aquellos estudiantes promedio, que si bien, no están atravesados por ninguna condición de vulnerabilidad, sus formas de aprender también sean visibilizadas por el profesor con el fin de ser atendidas y para que el aprendizaje no resulte comprometido?

Por tal motivo, el presente artículo pretende dar a conocer el trabajo que se viene realizando, en el campo de las concepciones de los educadores sobre la diversidad en relación con el aprendizaje escolar, para tener un panorama más claro de las diferentes posturas o abordajes que se enfocan en este tema.

\section{Metodología}

La investigación presenta un diseño cualitativo-interpretativo, de tipo documental, el cual definió el procedimiento de selección, y registro de la información.

En la investigación se seleccionaron 41 investigaciones, realizadas en los años 2005, 2008, 2010, 2012 y 2013; incluyendo: trabajos de investigación, de revisión teórica, artículos de reflexión y tesis doctorales. Se utilizaron bases de datos en español y en inglés, de la Universidad del Valle y la Universidad de San Buenaventura, tales como Scopus, Web of Science, Latindex, Publindex, Biblioteca de la ClaCso, TDR (Tesis Doctorales en Red); entre otros. Los descriptores utilizados fueron: concepciones, educador infantil, diversidad y aprendizaje escolar (conceptions, childhood educators, diversity, school learning). Para la escogencia de los documentos se usaron como criterios de selección el año de la publicación, la relevancia y pertenencia con el tema de estudio, y poseer abundante información, de tal forma que se lograra completar la matriz

\section{Instrumentos}

Se utilizó una matriz bibliográfica donde se clasificaron todos los documentos de acuerdo con unos atributos o categorías. En el sentido vertical se relacionaban los documentos del estudio y en el sentido horizontal las categorías, tal como lo presenta en la figura1.

\begin{tabular}{lllllll}
\hline NOMBre & TIPo & OBJETIVOS & REFERENTES & Método & RESUltados & ConClusiones \\
\hline
\end{tabular}

Figura 1. Matriz bibliográfica

Fuente: Elaboración propia. 


\section{Procedimiento}

Con la lectura de cada texto, se procedió a alimentar la matriz de acuerdo con la información solicitada. Las categorías de análisis son las que direccionan el proceso investigativo y van arrojando la información relevante para su posterior análisis, el cual permitió que emergieran unos focos y tendencias que se presentan a continuación:

\section{Resultados}

\subsection{Foco 1: Atribuciones de la diversidad}

Este foco hace referencia a las atribuciones que los educadores infantiles le asignan a la diversidad. Se encontraron 4 tendencias descritas a continuación.

Tendencia 1: Atribuir diversidad a la condición de estudiantes extranjeros e inmigrantes (35\%)

En Durand (2008) se plantea que, para trabajar la diversidad en la educación inicial, los docentes en sus prácticas educativas requieren salir de los márgenes tradicionales del plan de estudios, e infundir principios de diversidad, equidad y justicia social en los niños, niñas y sus familias.

La tesis doctoral de Louzano (2011), tuvo como objetivo principal elaborar un proyecto educativo intercultural destinado en una parte a comprender las ideas, expectativas y actitudes que tiene el educador en cuento a la presencia de población educativa extranjera. Los resultados arrojaron que más del $85 \%$ de los educadores valora positivamente compartir en el aula con niños y niñas de otros países, de otras culturas.

El estudio de Vassiliki Riga, Zampeta Papadodima y Konstantinos Ravanis (2012), indagó sobre las creencias de los futuros profesionales, acerca de sus capacidades de enseñanza en el ámbito de la educación preescolar multicultural. Como conclusión refieren la importancia de ofrecer a los futuros educadores infantiles la competencia y la capacidad de enseñanza intercultural, así como la posibilidad de sensibilizarlos en los temas de la educación equitativa para los niños y niñas independientemente de su origen e identidad.

Izquierdo (2004) afirma que los referentes sociales y culturales ayudan a contextualizar la realidad educativa del niño, pero no es el único indicador. Así mismo, Rodríguez (2005) refiere que la actitud y comportamiento del maestro, sus creencias y expectativas acerca de la capacidad y logro del estudiante, influye de manera determinante en la creación de un concepto académico positivo o negativo del alumno.

En otro trabajo de Izquierdo (2005) se investigó los factores relacionados con las actitudes y creencias de los estudiantes hacia la diversidad cultural y la Educación Intercultural. Los resultados arrojaron que las actitudes de los estudiantes de Magisterio son claramente favorables hacia la diversidad cultural. De igual forma, el estudio refiere la importancia de la formación de los maestros, en temas y exigencias de la realidad multicultural.

Gómez y Souto (2011) tenían como propósito constatar la relación entre la formación de maestros y maestras de educación infantil de acuerdo a su antigüedad, sus experiencias profesionales interculturales y su actitud ante el modelo de escolarización de hijos de inmigrantes. Como conclusión se expone la importancia de que en el pensum académico de los futuros educadores se incluyan materias que desarrollen las competencias docentes necesarias para una educación intercultural. 
El estudio de Cartón, Balerdi, Larrañaga y Aranguren (2012) en su metodología utilizaron un cuestionario que se aplicó a los educadores, familiares y responsables educativos sobre las competencias que consideraban más idóneas para los profesionales que trabajaban habitualmente con estudiante inmigrante. Los resultados arrojaron la importancia de impulsar actividades que supongan intercambio y relación entre alumnado inmigrante y autóctono; se recomienda evitar guetos y grupos separados.

En la tesis doctoral de Murray (2012) los objetivos fueron identificar cuáles eran los valores, las actitudes, conocimientos y estrategias de los profesionales y las familias para promover la integración social de los niños de diversos orígenes culturales en centros de desarrollo infantil. Los resultados demostraron la importancia del modelo de educación intercultural como la herramienta necesaria para hacer frente a los desafíos que los educadores enfrentan, tales como la promoción de la integración social, el respeto a la diversidad y el multilingüismo y la prevención de actitudes y acciones racistas, xenófobas y discriminatorias.

Ríos, Moledo y Rego (2014) examinaron la concepción de educación intercultural que subyace entre el profesorado de educación infantil y primaria. Los resultados demuestran que las percepciones de los docentes de educación infantil y primaria sobre la Educación Intercultural están dirigidas a armonizar la cultura de origen de los estudiantes con la adquisición de nuevas costumbres y valores en la nueva escuela.

Matencio, Molina y Miralles (2015) indagaron la percepción de los maestros de educación primaria sobre la educación intercultural, con la finalidad de anticipar si dicho profesorado está contribuyendo o no, a desarrollar la educación intercultural en sus centros educativos. Como resultado, se encontró que un $60 \%$ de los educadores no creen en la educación intercultural. De igual forma, se encontró que la diversidad cultural puede ser un obstáculo en la escuela, aunque puede tener solución si las actitudes de los educadores son positivas. En general, este estudio permite comprender que se continúa asociando la diversidad cultural con el fracaso escolar.

Guevara (2010) trabajó con una docente de preescolar, una niña y dos niños nicaragüenses del nivel de transición. El propósito de la investigación era analizar la relación entre la práctica docente de una maestra costarricense del nivel transición del jardín público Luces y las propuestas educativas para la atención de la diversidad cultural. Esta investigación permitió comprender que más allá de trabajar con los principios de la educación intercultural, los educadores asumen que dar un trato igualitario a todos los estudiantes es, de por sí, un logro valioso, aunque continúen con prácticas asimilacionistas y excluyentes.

La investigación de Novaro, Borton, Diez y Hecht (2008) presenta como conclusión que los educadores deben conocer y comprender primero las conductas propias de la cultura de los estudiantes inmigrantes y no anteponer o asumirlas como conductas agresivas o disruptivas. De esta manera las concepciones que los educadores tengan sobre diversidad facilitarán que estos niños se sientan seguros, respetados y visibilizados.

Kidd, Sánchez y Thorp (2005) presentaron los resultados de un programa de preparación de maestros de primera infancia que tuvo una duración de dos años y donde se examinaron los cambios en las disposiciones culturales mientras se relacionaban y convivían con niños de orígenes culturalmente diversos. La conclusión de este estudio versa sobre la importancia de la formación y el contacto por parte de los educadores, con niños y niñas 
de orígenes culturales y lingüísticos diversos, para poder cambiar las percepciones y actitudes en cuanto a ellos y sus familias. Esto favorece el diseño e implementación de un currículo más cercano que permita atender las necesidades individuales de los niños, así como valorar la continuidad entre el hogar y la escuela.

Como puede observarse, en las anteriores investigaciones la diversidad se configura a partir del reconocimiento y aceptación de la existencia de población estudiantil extranjera o inmigrante.

Tendencia 2: Atribuir diversidad a la condición de estudiantes en condición de discapacidad (25\%)

Aquí encontramos el estudio de Thornton y Underwood (2013) en el que se examinaron las concepciones sobre discapacidad desde la perspectiva de cuatro educadores infantiles. Este estudio invita a los profesionales a reflexionar de manera crítica sobre su práctica y a considerar sus creencias y comportamientos como factores claves en la creación y el mantenimiento de ambientes inclusivos de aprendizaje para todos los niños y niñas; pues de creer o pensar que la discapacidad es una desventaja o una limitación, las oportunidades del niño o niña discapacitado podrían reducirse.

La tesis doctoral de Gómez (2011) presenta como propósito analizar, describir, contrastar e interpretar la gestión de la diversidad y las prácticas directivas en los centros públicos de primera infancia. Como conclusión se encontró que hay equipos directivos que todavía asocian el concepto de diversidad al alumnado con necesidades educativas especiales. Esto promueve una actitud segregadora ante la diversidad, pues desde la gestión escolar se entenderá la diversidad como un déficit y no como un elemento enriquecedor.

Los resultados del estudio de San Martín (2012) identifican dificultades para llevar a cabo la tarea de atender la diversidad: falta de formación profesional y de tiempo, elevado número de alumnos por aula y desfase en el desarrollo de las habilidades de los alumnos que presentan discapacidad intelectual en relación a la clase.

Macartney y Morton (2013) plantean que las percepciones de los educadores sobre discapacidad continúan en el plano del déficit o la limitación. De esta manera, la diversidad va a tomarse desde un lugar negativo. Los resultados encontrados confirman que las percepciones se construyen y reconstruyen a través de la vida diaria en el aula de clase y van ganando mayor terreno. Por lo tanto, desconocer el contex to y asumir como problema a un estudiante, sin entenderlo en su totalidad, va a llevar a que las prácticas educativas sean sesgadas y se le atribuya a la diversidad una connotación de problema o limitación.

Messias, Muñoz y Torres (2013) describen y analizan el proceso de atención a la diversidad de un niño con discapacidad múltiple en un Centro de Educación pública de Educación Infantil y Primaria en Castilla-La Mancha, destacando los procesos de convivencia en el aula y en el aprendizaje. Los resultados refieren que los educadores manifestaron que sus creencias muchas veces se deben a que ignoran o desconocen la situación tal como es, y tener la oportunidad de estar cerca de la discapacidad hace que sus creencias cambien y se puedan ver cambios en sus prácticas educativas. Para ellos, la diversidad es entender que la discapacidad no es una limitación sino una oportunidad de aprendizaje que conlleva a unos resultados positivos.

Vázquez, Borzi y Talou (2013) trabajaron con respecto a las concepciones, creencias y conocimientos sobre la inclusión de niños con Necesidades Educativas Derivadas de las Discapacidades. Las conclusiones de este estudio plantean que los educadores infantiles 
presentan concepciones y creencias erróneas en torno a la discapacidad por la falta de preparación y conocimiento durante sus años de formación universitaria. Para ellos es necesario un cambio de paradigma y avanzar en el tema de producción de teorías que permitan un manejo de la discapacidad como diversidad viable y retadora para ellos.

Sánchez, Rojas, Soto y Perafán (2014) exploraron los imaginarios y referentes que poseen los educadores infantiles acerca de la atención educativa integral de la primera infancia con necesidades educativas especiales en Florencia (Caquetá). Los resultados arrojaron que el $59 \%$ de los educadores afirman que por falta de recursos, material didáctico, recursos audiovisuales e infraestructura institucional no se logra atender de manera integral a niños y niñas con NEE. El (14\%) de los educadores consideran que la falta de seguimiento y atención integral a los niños y niñas es un factor decisivo en la deficiente atención educativa.

Las conclusiones de la tesis doctoral de Bogéa (2012) refieren la urgente implementación de una política de formación docente como forma de desmitificar la inclusión escolar como una actividad solo de especialistas. De igual forma, utilizar los conocimientos teóricoprácticos para transformar sus prácticas y en la superación de la dicotomía entre la enseñanza regular y la especial. De esta manera, la discapacidad como diversidad podrá tener la connotación de posibilidad y no de limitación.

Entre las conclusiones de la tesis doctoral de Romero (2014) está la importancia por parte de los educadores de tener una visión igualitaria de los estudiantes, valorándolos y respetándolos como personas. Esto favorecerá efectuar adaptaciones y adecuaciones curriculares, implementar un currículo comprensivo, único y diverso, donde se respetan los tiempos y las diferencias individuales de cada niño y niña.

En la tesis doctoral de Meza (2010) donde se exploran las percepciones de los educadores, se menciona la falta de formación adecuada para atender a niños con necesidades educativas especiales, lo que conlleva a que sus prácticas no sean las más favorables. Por lo tanto, sus creencias y percepciones pueden verse afectadas y no aprovechar el aprendizaje colaborativo como una estrategia positiva en el aula de clase. No obstante, el análisis de las percepciones de los educadores en función de su grupo profesional de pertenencia reveló que una mayor formación se relaciona con percepciones y puntos de vista más positivos acerca de la inclusión.

Como puede observarse, en las anteriores investigaciones, la diversidad se configura, a partir del reconocimiento y aceptación de la existencia de población estudiantil en condición de discapacidad, es decir, una diversidad que denominan funcional. Ainscow (2005), considera que, si la diversidad se enfoca únicamente en los estudiantes que presentan una mayor problemática o carencia, es una mirada muy reduccionista. Desde esta mirada, una parte de población estudiantil se está invisibilizando.

Tendencia 3: Atribuir diversidad a la condición de estudiantes étnicos, específicamente población indígena (15\%)

El trabajo de Lynn Ang (2010) refiere que los educadores infantiles deben ser críticamente conscientes de su propia cultura macro y de cómo, incide en su comportamiento, rutinas de cuidado y, adicionalmente en su labor profesional como educador. Por lo tanto, el plan de estudio puede verse afectado por sus propios valores culturales, familiares e individuales. Finalmente, este estudio señala la necesidad de ofrecer un plan de estudios que facilite la comprensión de otros grupos étnicos, pero hace también el llamado a 
trabajar las relaciones interpersonales e intergrupales y la promoción de una creciente sensibilidad hacia las diferencias.

González, Sánchez y Araque (2013) hacen énfasis en los procesos transicionales que hacen los niños indígenas de sus escuelas étnicas a las escuelas de la ciudad en el sector oficial. $\mathrm{El}$ aporte de esta investigación señala la importancia de las actitudes de los educadores en pro de permitir que la identidad cultural del niño y niña no se pierda y, en vez de negarla, la utilicen como recurso para enseñar a los otros niños y niñas las diferencias.

Aguaded (2005) pretende analizar las competencias curriculares de los estudiantes a través de las propias actividades que realizan en las aulas, para proponer y realizar intervenciones acordes a la Educación Intercultural y evaluar sus consecuencias. Los resultados refieren que los educadores perciben la diversidad desde el déficit de aprendizaje que pueden presentar estos niños y niñas procedentes de población indígena. Por tanto, las clases se direccionan a incidir sobre el déficit y descuidan la integración social en el grupo. Esta mirada de la diversidad es muy reduccionista y restringe las posibilidades de que los niños y niñas tengan oportunidades.

Carpio (2015) pretendió conocer el discurso de los futuros educadores infantiles sobre diversidad cultural. Es una investigación cualitativa cuyo método para la recolección de la información fueron grupos de discusión. La conclusión principal del estudio refiere la falta de formación de los futuros educadores para enfrentar los retos de la educación intercultural. De ahí, la necesidad de implementar planes de estudio que valoren el trabajo en valores (sensibilidad, empatía, capacidad crítica, etc.); formación en la reflexión y orientada hacia conceptos, ideas y actitudes, más que en técnicas o metodologías y el desarrollo de capacidades propias de un mundo intercultural.

Guzmán, Cely y Salazar (2015) pretendían conocer a través de los relatos de los educadores su experiencia en la infancia con respecto a la vivencia con la diferencia y cómo esta experiencia influyó en su ejercicio profesional y cuánto ha favorecido en la construcción de propuestas pedagógicas más incluyentes. Las conclusiones refieren la importancia del diálogo y la escucha, como mecanismos para conocer y entender al otro. De igual forma, invita a pensar en el papel del educador, no como un simple transmisor de conocimiento, sino, como una persona abierta al diálogo.

Barriga (2015) pretendía brindar un panorama por las diferentes conceptualizaciones y discursos acerca de la diversidad y su impacto en el ámbito de la educación infantil, con el fin de brindar unas bases teóricas donde se promueva la reflexión sobre la escuela como espacio para la interacción dialógica. La conclusión principal apunta a referir que los niños y niñas que provienen de comunidades indígenas enfrentan dificultades debido a las tensiones entre la cultura dominante y la minoritaria.

Las anteriores investigaciones dan cuenta de la diversidad bajo una connotación de déficit o falta, pues se considera a la población indígena, como una población vulnerable, que presenta muchas necesidades y desventajas socioculturales. Lo anterior provoca una connotación en los imaginarios colectivos de deficiencia en la apropiación de los conocimientos (occidentales) en las poblaciones en mención. Lo anterior, deja ver la urgencia de deconstruir la educación homogenizante, de blanqueamiento hacia una educación intercultural donde se propende por la des-victimización de las etnias por el reconocimiento de sus saberes, de sus memorias, de sus usos y costumbres, esto es, de su 
cosmovisión desde una propuesta pedagógica y formativa en y para su pervivencia y visibilización.

Tendencia 4: Atribuir diversidad a toda la población educativa sin ninguna condición especifica (25\%)

El trabajo de Katia Gramelt (2013) presentó un programa para mejorar la capacidad de los educadores infantiles en el tema de la diversidad. Los resultados demuestran que, si bien los educadores dicen no presentar actitudes de rechazo con sus estudiantes, la población dominante (el estudiante promedio) presenta mayor ventaja que otros niños y niñas.

Roberts (2012) presenta las experiencias de los educadores infantiles sobre la implementación de un programa basado en el juego aplicado durante el año 2010. Como resultado se encontró la disposición y actitudes positivas de los educadores hacia el tema de la diversidad mediante el juego; no obstante, también se mencionan algunos impedimentos que demoran o interfieren con el trabajo de la diversidad, como son las exigencias en el manejo del tiempo, presiones, resultados inmediatos, entre otros.

Purduea y otros (2009) reflexionaron sobre la importancia de ir más allá de los enfoques tradicionales y ofrecer mayor teoría al respecto, de tal modo, que la inclusión alcance realmente su objetivo de atender a toda la población educativa, sin necesidad de presentar alguna condición específica. En este estudio las actitudes de los educadores infantiles son cruciales, pues son ellos los que permiten o impiden que la educación inclusiva logre su objetivo.

Warming (2009) también presenta una reflexión sobre la importancia de los discursos de los educadores infantiles a la hora del trabajo en el aula de clase. De las creencias que tengan los educadores infantiles, la diversidad en el aula se puede ver amenazada o realmente puede cumplir con su papel en la interacción social de todos los niños y niñas.

Petriwskyj (2010) destaca la falta de acceso a servicios de apoyo, la disponibilidad de recursos para la enseñanza, las presiones en los resultados y el aprendizaje profesional limitado como factores que entorpecen el trabajo de los educadores infantiles sobre la diversidad en el ambiente de aprendizaje. En esta investigación se habla del estudiante diverso y destaca el cambio pedagógico como una prioridad clave para la respuesta eficaz a la diversidad.

Lebrero (2008) refiere que los educadores conciben el término atención a la diversidad para todo el alumnado, y lo definen como un modelo de educación: intercultural, integrador, compensador de desigualdades, con enfoque de género y para la cohesión social. Refieren que progresivamente se amplía la necesidad de integrar a niños con características diferentes respecto a los mal denominados normales, debido al aumento de la sensibilización social y educativa frente a las desigualdades, y en reconocimiento de los derechos de todo ser humano a disponer del máximo de oportunidades.

Blanco (2009) plantea que la educación inclusiva implica una visión diferente de los educadores basada en la diversidad y no en la homogeneidad, considerando que cada estudiante presenta unas capacidades, intereses, motivaciones y experiencia personal única, es decir, las diferencias son inherentes a los seres humanos y por lo tanto están dentro de lo esperado. 
Guijo (2008) reflexiona sobre cómo la escuela inclusiva, y en especial el rol de los educadores, puede atender en la etapa de educación infantil los derechos de la infancia al propiciar que todos los niños y niñas aprendan juntos con independencia de sus condiciones personales, sociales o culturales. Se analiza cómo la educación inclusiva debe tener como objetivos disminuir el riesgo de exclusión social, a la vez que se ha de propiciar el desarrollo de la personalidad, las aptitudes y la capacidad mental y física del menor hasta el máximo de sus posibilidades. Esta educación ha de contribuir a los aprendizajes básicos que aseguren la integración y el éxito escolar.

García (2013) propone un concepto: las Necesidades Educativas Personales (N.E.P.). Este término no agrupa únicamente las necesidades educativas de aquellos alumnos que más dificultades encuentran en el proceso de enseñanza-aprendizaje, sino a todo alumno que se halle inmerso en él, sea cual sea la intensidad de estas. Debe ponerse el énfasis en las capacidades de cada alumno, y no en las carencias y/o dificultades que presente. De igual forma refiere que la formación de los educadores debe llevar a que sus creencias y prácticas desarrollen al máximo el potencial individual de cada niño, fomentando la equidad, la justicia social, y la inclusión en los centros educativos para que todos los niños reciban el tratamiento educativo que se merecen.

González, Rubio y Rubio (2014) demuestran la existencia de limitaciones en el abordaje del tema de la atención a la diversidad en la infancia preescolar, que afectan el logro del desarrollo pleno de los niños en esta etapa. De igual forma, consideran necesario la necesidad de implementar un modelo pedagógico por parte de los educadores infantiles fundamentado en una perspectiva preventiva e inclusiva que tome como criterio esencial la individualidad de todos y de cada uno los niños, con énfasis en las relaciones que se deben establecer entre lo cognitivo, lo instrumental y motivacional para lograr un desarrollo más pleno e integral de los niños y niñas.

Parrado (2015) explora la importancia del reconocimiento de la diversidad y su relación con población estudiantil considerada normal, con capacidades superiores o diversidad funcional, permitiendo innovar la formación infantil bajo una mirada inclusiva. Los resultados arrojaron que las representaciones sociales sobre diversidad en los estudiantes, docentes y egresados del programa de Licenciatura en Pedagogía Infantil se construyen desde el reconocimiento de la diferencia de los otros y el papel que desempeña la cultura en la formación de la identidad de la persona.

Como se puede apreciar, el porcentaje de investigaciones que trabajan la diversidad como escenario para todos los estudiantes en contextos considerados no diversos o específicos sigue siendo bajo con respecto a los otros estudios. De ahí la importancia de visibilizar aquello que está oculto o relegado. La oportunidad del reconocimiento de las diversidades puede ser para todos; este es un tema de alta relevancia, porque implica la visibilidad de todos y cada uno de los niños y niñas en los diversos contextos educativos. De Sousa (2006) refiere la expresión sociología de las ausencias para referirse a la necesidad de tematizar aspectos invisibilizados por el orden hegemónico. Estas investigaciones nos muestran que se sigue manejando un concepto de diversidad muy sesgado; pensar una idea de diversidad más amplia implica conocer cuáles son los discursos hegemónicos que la han atravesado y cómo eso afecta las atribuciones que se hacen a nivel educativo. En esta línea, la diversidad que implica igualdad de oportunidad para todos no puede estar en el orden de lo hegemónico y de lo excluyente. Por lo tanto, es importante indagar las concepciones de los educadores infantiles en relación a la diversidad y su afectación en el aprendizaje 
escolar de los estudiantes, para que de esta manera se pueda construir una mirada más amplia de diversidad que abarque a todos los niños y niñas del salón de clase, sin ninguna excepción.

\subsection{Foco 2: Aspectos metodológicos de la investigación}

Un aspecto importante al analizar los estudios sobre las concepciones de los educadores sobre la diversidad es el proceso de investigación abordado. Se exponen las líneas generales de la ruta metodológica que siguieron para alcanzar estos conocimientos.

En este foco se identificaron 5 tendencias, la principal, con un $96.6 \%$ de las investigaciones, se llevaron a cabo con metodologías cualitativas, con la cual se estudian los hechos en sus ambientes reales, intentando darles sentido e interpretando los fenómenos en función de los significados que las personas les otorgan (Creswell, 2007). En este caso, lo que se pretendía estudiar eran las vivencias y creencias de los educadores con respecto al trabajo de la diversidad en el aula.

Es importante destacar que muy pocos estudios presentaron la información metodológica completa. Algunos presentaban solo el diseño, otros los métodos y otros los instrumentos. No obstante, se organiza la información recolectada en unas tendencias metodológicas y los porcentajes asignados son de manera proporcional.

\section{Tendencia 1: Propósitos investigativos}

Esta tendencia presenta las intenciones o fines de la investigación.

- Investigaciones Exploratorias: $41 \%$. Estudios que pretenden descubrir y evidenciar fenómenos o situaciones en un contexto determinado.

- Investigaciones Descriptivas: 33\%. Estudios que pretenden representar la realidad y dar cuenta de una situación especial bajo un contexto específico.

- Investigaciones Propositivas: 26\%. Estudios que pretenden transformar la realidad a través de aportes o propuestas para solucionar problemas.

Tendencia 2: Métodos investigativos

Esta tendencia presenta la forma de abordar el objeto de estudio. El método se elige de acuerdo a la naturaleza del problema y su forma de investigarla. Cada uno brinda la mejor posibilidad para recoger la información.

- Etnografía (42\%). Abordaje de las vivencias y creencias desde la perspectiva de los actores mismos, en este caso de los educadores infantiles.

- Estudios de caso (8\%). Exploración particular y exhaustiva de las concepciones de uno o varios educadores.

- Biográfico-narrativo (17\%). Reflexiones sobre las vivencias de los propios educadores.

- Teoría fundada (8\%). Generar teoría a partir de las experiencias con los educadores.

- Trabajos basados en métodos mixtos, cartografías, evocación de palabras (25\%). 
Tendencia 3: Instrumentos de recolección de la información

Esta tendencia presenta las técnicas utilizadas para la recolección de la información.

- Entrevistas (30\%). Conversación entre dos o varias personas, a través de unas preguntas estructuradas o semiestructuradas para indagar o comprender alguna situación o evento.

- Observación participante (24\%). Se exploran y describen los ambientes o situaciones de manera detallada.

- Cuestionario (14\%). Se presentan dos tipos: los estandarizados, que por lo general son instrumentos como escalas, cuyas respuestas son de tipo cerrada; y los flexibles, de elaboración propia a partir de los conceptos básicos de partida y las variables consideradas (Carpio, 2015).

- Encuestas (19\%). Instrumentos estandarizados como pruebas o test, o en forma de preguntas abiertas, que pretenden explorar y medir una situación.

- Análisis documental (10\%). Revisión de información contenida en libros, informes, cartas, legislación; que dan cuenta del fenómeno o situación a estudiar.

- Grupos de discusión (3\%). Encuentro de varias personas, a través del cual se propone un tema específico con el fin de explorar las creencias u opiniones sobre algún evento o situación.

Se aprecia que en estas investigaciones existe escases de trabajos con grupos de discusión como técnica de recolección de información, en tanto implican dar lugar a la palabra, queriendo conocer en lo más profundo, los deseos, miedos y reflexiones de las personas, frente a lo que se quiere indagar, lo que exige mayor dedicación del investigador. Por esta razón, algunos de ellos, prefieren técnicas más precisas y menos abiertas, lo que les implica menos tiempo y mayor facilidad para el análisis de las categorías.

De igual forma, puede observarse que las investigaciones sobre concepciones o creencias se trabajan principalmente desde lo etnográfico, esto queriendo reflejar la importancia de conocer el contexto y más aún, de interactuar con las personas interesadas en el estudio.

\subsection{Foco 3: Nombres con los que se designa el pensamiento de los educadores}

Al revisar los estudios sobre las concepciones de diversidad se encontró que si bien es cierto que el título de algunas investigaciones incluía algunas de las palabras descritas a continuación (creencia, percepciones, etc.), se pudo observar que, en el desarrollo del estudio, especialmente en los referentes teóricos o en los mismos resultados, ya no se hablaba de la misma palabra, sino que se hacía referencia a otra (actitud, representación, etc.), es decir, como si fueran sinónimos. Como ejemplo tenemos la investigación de Riga, Papadodima y Ravanis (2012) cuyo título es Percepciones de futuros maestros sobre las materias didácticas en el jardín de infantes multicultural como contexto educativo para los hijos de migrantes en Grecia, pero en todo el documento se habla es de creencias. Otro caso se presentó cuando en el titulo o propósito de la investigación se hacía referencia a la palabra imaginario, concepción, etc.; sin desarrollarlo en los referentes teóricos. Por lo tanto, no hubo claridad en la precisión de los términos. No obstante, las siguientes 
tendencias se sacan con base en la palabra que más énfasis se hizo en el texto y se define a partir de lo que se conceptualizó.

- Tendencia 1, Trabajos que hablan de Creencias (38\%). Son conocimientos subjetivos, de poca elaboración y producidos a nivel individual para justificar acciones y decisiones (Moreno, 2000).

- Tendencia 2, Trabajos que hablan de Actitudes (18\%). Rodríguez (1991) definió la actitud como una organización duradera de creencias y cogniciones en general, dotada de una carga afectiva a favor o en contra de un objeto definido, que predispone a una acción coherente con las cogniciones y afectos relativos a dicho objeto. Toda actitud posee un indiscutible componente cognitivoexperiencial (Currás y Dosil, 2001).

- Tendencia 3, Trabajos que hablan de Concepciones (18\%). Son cuadros conceptuales o referentes teóricos sobre algún fenómeno o situación (Ponte, 1994).

- Tendencia 4, Trabajos que hablan de Percepciones (12\%). Es la comprensión e interpretación de la realidad de un fenómeno o situación.

- Tendencia 5, Trabajos que hablan de Imaginarios (2\%).

- Tendencia 6, Trabajos que hablan de Representaciones Sociales (2\%). Conjunto de interpretaciones que un grupo realiza sobre algún aspecto de la realidad y que, a su vez, guían las acciones correspondientes (Moscovici, 1961).

Estas investigaciones enuncian que el estudio trata sobre concepciones, creencias, percepciones o representaciones de los educadores, pero muy pocos los desarrollan teóricamente para dar una claridad sobre el concepto. De igual forma, en los estudios no se evidencia el origen, causa o factor que desencadena esa creencia o concepción, lo cual hace que la investigación pierda peso cuando lo enuncia y no lo desarrolla. Esto alude más bien a una valoración de la calidad de la investigación no refleja una tensión.

\section{Discusión}

El estudio permite evidenciar una primera tensión que versa sobre la poca claridad que todavía existe sobre la concepción de diversidad. Aun se asocia con situaciones o condiciones específicas bajo una connotación de déficit o vulnerabilidad.

Como segunda tensión se encuentra la confusión en la palabra diversidad y más aún en la palabra concepción. Es decir, hay una doble confusión lo que dificulta su compresión como concepto. Diversidad, por estar ligada a varias connotaciones y la palabra concepción, por ser una construcción a partir de creencias y muchas veces lleva implícito un valor, que entra a interferir en la definición del concepto. La tercera tensión que se encuentra en esta revisión es la crítica que se le hace a la cultura de lo homogéneo como oposición a la diversidad. Igualmente se explicita el reconocimiento a lo étnico como diversidad, siendo claros que al interior de esta cultura étnica también hay homogeneidad. Una cuarta tensión se encuentra en el discurso del reconocimiento de la diversidad de niños y niñas en el aula de clase y en las escuelas, y otra muy distinta es la escenificación de este discurso en la práctica. Se observa que, en lugar de promover el respeto a esas diferencias, se hace énfasis en una educación homogenizante para niños con características iguales. 


\section{Conclusiones}

Se puede inferir que el estado del arte construido hace referencia a una diversidad instituida entendida como aquello que el común denomina, clasifica y determina y no así, como una diversidad instituyente que precisamente emerge de las particularidades y cambios de una sociedad.

El discurso de la diversidad como posibilidad para todos está lejano de ser una realidad (Rodríguez, 2005). Como puede verse, la mayoría de las investigaciones revisadas, demuestran que la diversidad aún se concibe como característica especial de alguna situación o condición; y lo más crítico todavía, es que se asocia a déficit o tiene una connotación de limitación.

En cuanto a los instrumentos de recolección de la información, la observación participante y las entrevistas son las más utilizadas para indagar las concepciones de los educadores, pues estas permiten dar cuenta de la práctica y que hacer del educador. Así mismo, las investigaciones sobre concepciones o creencias se trabajan principalmente desde lo etnográfico, dando a conocer el contexto y más aún, de interactuar con las personas interesadas en el estudio. De ahí que las entrevistas narrativas en el campo del estudio de las concepciones tengan mayor interés.

Se encuentra poca claridad en los términos de creencias, concepciones y percepciones de los educadores. Se trabaja más con creencias, lo que sugiere que no hay tanto conocimiento y formulación de conceptos en este campo de estudio de la diversidad en la educación inicial y su implicación en el aprendizaje escolar.

Se hace necesario reflexionar y aportar teoría en este campo de la diversidad, donde se responda a la particularidad de cada sujeto, no centrándose en la falta o carencia; sino, bajo una mirada que integre toda la persona, reconociéndola como única e irrepetible. De ahí, que sea pertinente, conocer como ha sido el proceso de construcción de esta palabra y como la historia de vida de los educadores ha incidido en que estos la asuman como tal. Esta teoría debe partir del mismo contexto local, en el mismo que hacer de los educadores en su contacto con los niños, que dé cuenta de su realidad y de como la llaman o enuncian en su día a día.

Finalmente, el reconocimiento de diversidades -otras, dentro del procesos educativo y formativo conlleva a reconocer el camino formativo como un camino de ir y venir, de contingencias, de reconocimiento de saberes otros, de saberes locales; esto es de voces silenciadas independientemente de su condición económica, étnica de género, religión, minoritaria o mayoritaria. Pensarse en la diversidad desde la singularidad del otro, de los otros lo que provoca una formación que dinamice las interacciones grupales, personales, los eventos comunicativos y el reconocimiento de los seres que cohabitan un espacio como únicos e irrepetibles. Así, la formación desde la diversidad busca el afuera para el adentro con voces de siembra y legado con grandes legados simbólicos que permitan continuar el tejido de les sabidurías, de las construcciones colectivas y de la reciprocidad.

\section{Referencias}

Aguaded, E. (2005). Diagnóstico basado en el currículum intercultural de aulas multiculturales en educación obligatoria. Tesis doctoral. Universidad de Granada. 
Ainscow, M. (2005, mayo). El próximo gran reto: La mejora de la escuela inclusiva. Ponencia presentada en el Congreso sobre Eficacia y Mejora Escolar. Barcelona.

Ang, L. (2010). Critical perspectives on cultural diversity in early childhood: Building an inclusive

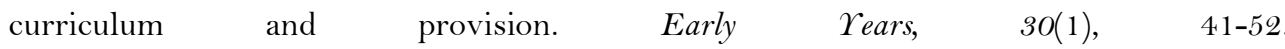
https://doi.org/10.1080/09575140903562387

Barriga, A. M. (2015). Diversidad cultural en la primera infancia: Un reto educativo en contextos urbanos. Educación y Ciudad, 24, 119-132.

Blanco, R. (2009). La atención educativa a la diversidad: Las escuelas inclusivas. Calidad, Equidad y Reformas en la Enseñanza, 87, 1-10

Bogéa, M. A. O. (2012). Inclusión educativa de las personas con deficiencia en el contexto de las escuelas públicas del Estado de Maranhão: Políticas y prácticas. Tesis doctoral. Universidad de Alcala. España.

Bravo, A. (1999). Curriculum y educación intercultural elaboración y aplicación de un programa de educación intercultural. Tesis doctoral. Universidad de Lleida.

Carpio, C. (2015). Discurso de futuros docentes sobre de la diversidad intercultural. Revista de Sociología, 10O(2), 155-172. https://doi.org/10.5565/rev/papers.2086

Cartón, H. M., Balerdi, F. E., Larrañaga, J. G. y Aranguren, E. A. (2012). ¿Qué otras competencias debe tener el profesorado del alumnado inmigrante? Magis, 5(10), 1-24.

Creswell, J. W. (2007). Qualitative inquiry and research design: Choosing among five traditions. Nueva York, NY: Sage Publications.

Currás, C. y Dosil, A. (2001). Diccionario de psicología y educación. Santiago de Compostela: Xunta de Galicia.

De Sousa Santos, B. (2006). La sociología de las ausencias y la sociología de las emergencias: Para una ecología de saberes. B. De Sousa Santos (Ed.), Renovar la teoría crítica y reinventar la emancipación social (pp. 13-41). Buenos Aires: CLACSO.

Durand, T. M. (2010). Celebrating diversity in early care and education settings: Moving beyond the margins. Early Child Development and Care, 180(7), 835-848. https://doi.org/10.1080/03004430802466226

Dussel, E. (1977). Introducción a una filosofía de la liberación latinoamericana. Ciudad de México: Editorial Extemporáneos.

García Barrera, A. (2013). Proponiendo un concepto nuclear latente en educación: Las necesidades educativas personales (NEP). Tesis doctoral. Universidad Autónoma de Madrid.

Gimeno, (2000). La construcción del discurso acerca de la diversidad y sus prácticas. Aula de Innovación Educativa, 81, 67-72.

Gómez, C. I. O. y Souto, B. C. (2011). Formación, experiencia docente y actitudes de los profesores de infantil y primaria ante la educación escolar de hijos de personas inmigrantes en España. Revista de Investigación Educativa, 30(1), 111-130.

Gómez, I. (2011). Dirección escolar y atención a la diversidad: Rutas para el desarrollo de una escuela para todos. Tesis doctoral. Universidad de Huelva.

González, G. D. G., Sánchez, B. Y. L. y Araque, C. S. (2013). Procesos transicionales en niños y niñas indígenas de primera infancia, del distrito capital a su ingreso a la escuela pública. Revista ALETHEIA, 5(1), 16-33. 
González, I. C. G., Rubio, B. N. M. y Rubio, J. M. (2014). Fundamentos de un modelo pedagógico inclusivo y preventivo para la atención a la diversidad educativa en el grado preescolar. Innovación Tecnológica, 20, 1-24.

Gramelt, K. (2013). Diversity in early childhood education: A German perspective. Early Years, 33(1), 45-58. https://doi.org/10.1080/09575146.2012.677948

Guevara, L. S. R. (2010). Creencias, práctica docente y propuestas para la atención de la diversidad cultural: El caso de una docente costarricense y tres infantes nicaragüenses. Revista Electrónica Educare, 14(2), 155-172.

Guijo, V. (2008). Derechos de la infancia (O-6 años) y educación inclusiva. Revista de Educación, 347, $55-74$.

Guzmán, B. S., Cely, I. S. D. y Salazar, L. M. P. (2015). Voz y experiencia: Narrativas de maestros sobre la diferencia cultural. Revista Colombiana de Educación, 69, 205-222. https://doi.org/10.17227/01203916.69rce205.222

Izquierdo, R. M. R. (2005). Estudio de las concepciones de los estudiantes de magisterio sobre la diversidad cultural. Revista Educar, 36, 49-69.

Kidd, J. K., Sánchez, S. Y. y Thorp, E. K. (2005). Cracking the challenge of changing dispositions: Changing hearts and minds through stories, narratives, and direct cultural interactions. Journal of Early Childhood Teacher Education, 26(4), 347-359. https://doi.org/10.1080/10901020500413304

Lebrero Baena, M. P. (2008). Presentation. Sociocultural attention to the rights of early childhood. Revista de Educación, 347, 15-31.

Louzano, M. (2011). Elaboración y puesta en práctica de un proyecto escolar de educación intercultural en el marco de una investigación cualitativo-etnográfica. Revista Interuniversitaria de Investigación sobre Discapacidad e Interculturalidad, 5(1), 87-100.

Macartney, B. y Morton, M. (2013). Kinds of participation: Teacher and special education perceptions and practices of 'inclusion' in early childhood and primary school settings. International Journal of Inclusive Education, 17(8), 776-792. https://doi.org/10.1080/13603116.2011.602529

Matencio-López, R. M., Molina-Saorín, J. y Miralles-Martínez, P. (2015). Percepción del profesorado sobre concepciones profesionales ante la diversidad cultural escolar. Convergencia, 22(67), 181-210. https://doi.org/10.29101/crcs.v0i67.2187

Melero, M. L. (2004). Construyendo una escuela sin exclusiones: una forma de trabajar en el aula con proyectos de investigación. Madrid: Editorial Aljibe.

Messías, V. L., Muñoz, Y. y Torres, S. L. (2013). Apoyando la inclusión educativa: un estudio de caso sobre aprendizaje y convivencia en la educación infantil en Castilla-La Mancha. Revista Latinoamericana de Educación Inclusiva, 6(2), 25-42.

Meza García, C. L. (2010). Cultura escolar inclusiva en educación infantil: Percepciones de profesionales y padres. Tesis doctoral. Universidad de Salamanca.

Moscovici, S. (1961). El campo de la psicología social. Psicología social. Barcelona: Paidós.

Murray, J. (2012). Learning to live together: An exploration and analysis of managing cultural diversity in ten early childhood development centres in South Africa. Tesis Doctoral. Universidad Autónoma de Barcelona.

Novaro, G., Borton, L., Diez, M. L. y Hecht, A. C. (2008). Sonidos del silencio, voces silenciadas: niños indígenas y migrantes en escuelas de Buenos Aires. Revista Mexicana de Investigación Educativa, 13(36), 173-201. 
Ocampo, C. y Cid Suoto, B. (2012). Formación, experiencia docente y actitudes de los profesores de infantil y primaria ante la educación escolar de hijos de personas inmigrantes en España. Revista de Investigación Educativa, 1, 111-130.

Parrado, Z. (2015). Representaciones sociales de diversidad en el programa pedagogía infantil de la Universidad de los Llanos. Impetus, 10(2), 1-24.

Parrilla, A. (2006). Conceptualizaciones de la diversidad y diversidad de respuestas educativas. En J. Zardel, E. Adame y A. Ortiz (Comps.), Sujeto, educación especial e integración: Investigación, prácticas y propuestas curriculares. Ciudad de México: UNAM.

Petriwskyj, A. (2010). Diversity and inclusion in the early years. International Journal of Inclusive Education, 14(2), 195-212. https://doi.org/10.1080/13603110802504515

Ponte, J. P. (1994). Mathematics teacher's professional knowledge. Proceedings of the XVIII International Conference for the Psychology of Mathematics Education, 1, 195-210.

Purdue, K., Gordon-Burns, D., Gunn, A., Madden, B. y Surtees, N. (2009). Supporting inclusion in early childhood settings: Some possibilities and problems for teacher education. International Journal of Inclusive Education, 13(8), 805-815. https://doi.org/10.1080/13603110802110743

Riga, V., Papadodima, Z. y Ravanis, K. (2012). Percepciones de futuros maestros sobre las materias didácticas en el jardín de infantes multicultural como contexto educativo para los hijos de migrantes en Grecia. Miradas en Movimiento, 7, 26-47.

Ríos, F. X. C., Moledo, M. D. M. L. y Rego, M. A. S. (2014). La perspectiva del profesorado de educación infantil y primaria acerca de la educación intercultural. Magister, 26(2), 59-66. https://doi.org/10.1016/SO212-6796(14)70019-2

Roberts-Holmes, G. (2012). It's the bread and butter of our practice: Experiencing the early year's foundation stage. International Journal of Early Tears Education, 20(1), 30-42. https://doi.org/10.1080/09669760.2012.664473

Rodríguez, A. (1991). Psicología social. Ciudad de México: Trillas.

Rodríguez, R. (2005, enero). Éxito y fracaso escolar en contextos socioculturales interculturales: el reto de educar a estudiantes de diverso origen lingüístico y cultural. Comunicación presentada en el II Congreso Anual sobre Fracaso Escolar. Palma de Mallorca.

Romero, R. (2009). Modelo venezolano de integración educativa. Tesis doctoral. Universidad del Zulia.

Sánchez, A. C. T., Rojas, M. E. N., Soto, N. L. y Perafán, A. P. (2014). Inclusión escolar de primera infancia con necesidades educativas especiales: Imaginarios de los docentes. Infancias Imágenes, $11(2), 27-30$.

San Martín, C. (2012). Atención de la diversidad en el contexto educativo chileno: Concepciones del profesorado sobre evaluación y diseño de la propuesta curricular. REICE. Revista Iberoamericana sobre Calidad, Eficacia y Cambio en Educación, 10(4), 165-183.

Thornton, C. y Underwood, K. (2013). Conceptualisations of disability and inclusion: Perspectives of educators of young children. Early rears, 33(1), 59-73. https://doi.org/10.1080/09575146.2012.682975

Vázquez, M. J. S., Borzi, S. L. y Talou, C. L. (2013). La inclusión escolar en la infancia temprana: Convención de los derechos del niño a la sala de clase. Infancias Imágenes, 11(1), 41-48.

Warning, H. (2011). Inclusive discourses in early childhood education? International Journal of Inclusive Education, 15(2), 233-247. 


\section{Breve CV de las autoras}

\section{Angélica María Barrero}

Psicóloga de la Universidad del Valle. Especialista en la Enseñanza para la Educación Superior de la universidad Santiago de Cali. Maestría en Psicopedagogía Clínica. Estudiante VIII semestre del Doctorado en Educación énfasis en Estudios Culturales y Pensamiento Pedagógico Latinoamericano de la Universidad de San Buenaventura-Cali. Directora Centro NeuroPsicopedagógico Sentir \& Pensar. Miembro del grupo de investigación DPIE y de su línea de investigación en aprendizaje y desarrollo Universidad Santiago de Cali. Coordinadora área psicología educativa de la universidad Libre- Seccional Cali. ORCID ID: 0000-0003-3775-0983. Email: angelicambarrerop@unilibre.edu.co

\section{Ana Lucia Rosero}

Doctora en Ciencias Sociales, Niñez y Juventud del Centro de Investigaciones y estudios avanzados en niñez, juventud, educación y desarrollo de la alianza CINDE y Universidad de Manizales. Magister en Educación Especial. Énfasis en Dificultades en el Aprendizaje, Esp. en Docencia Universitaria, Licenciada en Filosofía y letras, Licenciada en Educación Preescolar. Miembro del grupo de investigación "Educación y pedagogía: saberes, imaginarios e intersubjetividades" y de su línea de investigación "Educación y Pedagogía”. Miembro del grupo Educación y desarrollo humano, línea Infancia Univ San Buenaventura Cali, Maestra-Investigadora Universidad de San Buenaventura, Cali. ORCID ID: 0000-0002-9364-925X. Email: alrosero@usbcali.edu.co 